\title{
Measurements of Two-Particle Correlations in pp collisions
}

\author{
Stefano Lacaprara* \\ on behalf of CMS collaboration \\ INFN Padova \\ E-mail: stefano.lacaprara@pd.infn.it
}

\begin{abstract}
We present results on two-particle angular correlations in proton-proton collisions over a broad range of pseudorapidity $(\Delta \eta)$ and azimuthal angle $(\Delta \phi)$. The data were collected with the CMS detector at the LHC. A complex two-dimensional correlation structure in $\Delta \eta$ and $\Delta \phi$ is observed. In the context of an independent cluster model of short-range correlations, the cluster size and its decay width are extracted from the two-particle pseudorapidity correlation function and compared with previous measurements in proton-proton and proton-antiproton collisions, as well as PYTHIA predictions. The results at $0.9 \mathrm{TeV}$ are in agreement with previous measurements. The new results at 2.36 and $7 \mathrm{TeV}$ represent the highest-energy measurements at a particle collider to date. Furthermore, Bose-Einstein correlations have been measured using samples of protonproton collisions at 0.9 and $2.36 \mathrm{TeV}$ center-of-mass energy. The signal is observed in the form of an enhancement of pairs of same-sign charged particles with small relative four-momentum. A significant increase of the size of the correlated particle emission region with the particle multiplicity in the event is observed.
\end{abstract}

35th International Conference of High Energy Physics - ICHEP2010,

July 22-28, 2010

Paris France

${ }^{*}$ Speaker. 


\section{Introduction}

This report gives an overview of the first results on two particle correlations obtained by the Compact Muon Solenoid (CMS) experiment with the data collected during the first months of the Large Hadron Collider (LHC) operations.

A detailed description of CMS detector can be found elsewhere [1]. The central feature of the CMS apparatus is a superconducting solenoid of $6 \mathrm{~m}$ internal diameter, providing a uniform magnetic field of $3.8 \mathrm{~T}$. The inner tracking system is the most relevant detector for the first analyses. It is composed of a pixel detector with three barrel layers at radii between 4.4 and $10.2 \mathrm{~cm}$ and a silicon strip tracker with 10 barrel detection layers extending outwards to a radius of $1.1 \mathrm{~m}$. Each system is completed by two endcaps, extending the acceptance up to a pseudorapidity $|\eta|=2.5$. Three detector sub-systems are used to trigger the detector readout for the data presented here. These are the forward hadron calorimeter (HF), the Beam Scintillator Counters (BSC) and the Beam Pick-up Timing for eXperiments (BPTX). The HF covers the region $2.9<|\eta|<5.2$, the two BSCs, located on either side of the IP at a distance of $\approx 11 \mathrm{~m}$, are sensitive in the range $3.23<|\eta|<4.65$. The two BPTXs are located around the beam pipe at $175 \mathrm{~m}$ from the IP and provide precise information on the structure and timing of the LHC beams.

\section{Two-Particle Angular Correlations}

Studies of multiparticle correlations have proven to be a powerful tool in exploring the underlying mechanism of particle production in high energy hadronic collisions. Proton-proton collisions, collected with a minimum bias trigger at $0.9,2.36$, and $7 \mathrm{TeV}$ center-of-mass energies, were used to study short-range correlations $(|\Delta \eta|$ less than $\approx 2)[2]$.

The $p_{t}$ - inclusive charged two-particle correlation function is defined as

$$
R(\Delta \eta, \Delta \phi)=\left\langle(N-1)\left(\frac{S_{N}(\Delta \eta, \Delta \phi)}{B_{N}(\Delta \eta, \Delta \phi)}-1\right)\right\rangle_{N}
$$

where $N$ represents the total track multiplicity of each event, $\Delta \eta=\left(\eta_{1}-\eta_{2}\right)$ and $\Delta \phi=$ $\left(\phi_{1}-\phi_{2}\right)$ are the relative difference in pseudorapidity and azimuthal angle between two particles. At a fixed multiplicity bin, the signal distribution $S_{N}(\Delta \eta, \Delta \phi)$ is the charged two-particle pair density function (normalized to unit integral). It is determined by taking particle pairs within the same event, then averaging over all events. The background distribution $B_{N}(\Delta \eta, \Delta \phi)$ denotes the distribution of uncorrelated particle pairs (normalized to unit integral). It is constructed by randomly selecting two different events from the same multiplicity bin and pairing every particle from one event with the other event, representing a product of two single particle distributions. The 2-D correlation at $\sqrt{s}=7 \mathrm{TeV}$ is shown in Fig. 1

To obtain quantitative results the 2-D correlation are projected into a 1-D pseudorapidity correlation function of $\Delta \eta$ and two different near-side and away-side $\Delta \phi$ ranges $(0<\Delta \phi<\pi / 2$ and $\pi / 2<\Delta \phi<\pi$ respectively).

In the context of an independent cluster emission description [3], $R(\Delta \eta)$ takes the functional form: $R(\Delta \eta)=K_{e f f}\left[\frac{\Gamma(\Delta \eta)}{B(\Delta \eta)}\right]$ where the correlation strength $K_{\text {eff }}$ is the effective cluster size. The 
function $\Gamma(\Delta \eta)$ is a Gaussian function $\propto \exp \left[-(\Delta \eta)^{2} /\left(4 \delta^{2}\right)\right]$ characterizing the correlation of particles originating from a single cluster where $\delta$ indicates the decay width of the clusters. Finally, $B(\Delta \eta)$ is the background distribution described above averaged over all multiplicity bins.

Fig. 2 shows the results of $K_{\text {eff }}$ and $\delta$ measured by the CMS experiment after the extrapolation to $|\eta|<3$ and $p_{t} \approx 0$, as well as previous measurements at lower energies and in the same pseudorapidity coverage. The extrapolated CMS results are $K_{\text {eff }}=2.50 \pm 0.01$ (stat) \pm 0.06 (syst) \pm 0.07 (extrap.), $\delta=0.64 \pm 0.01$ (stat) \pm 0.02 (syst) \pm 0.03 (extrap.) for $\sqrt{s}=0.9 \mathrm{TeV}, K_{\text {eff }}=2.65 \pm 0.03$ (stat) \pm 0.07 (syst) \pm 0.08 (extrap.), $\delta=0.60 \pm 0.01$ (stat) \pm 0.02 (syst) \pm 0.03 (extrap.) for $\sqrt{s}$ $=2.36 \mathrm{TeV}$, and $K_{\text {eff }}=2.75 \pm 0.01$ (stat) \pm 0.07 (syst) \pm 0.09 (extrap.), $\delta=0.59 \pm 0.01$ (stat) \pm 0.02 (syst) \pm 0.03 (extrap.) for $\sqrt{s}=7 \mathrm{TeV}$. The error bars in Fig. 2 include systematic uncertainties from both the experimental measurements and the extrapolations added in quadrature. The Pyтнia event generator shows a similar energy dependence of $K_{\text {eff }}$ and $\delta$ to the data, but essentially underestimate the magnitude of $K_{e f f}$ over the full energy range.
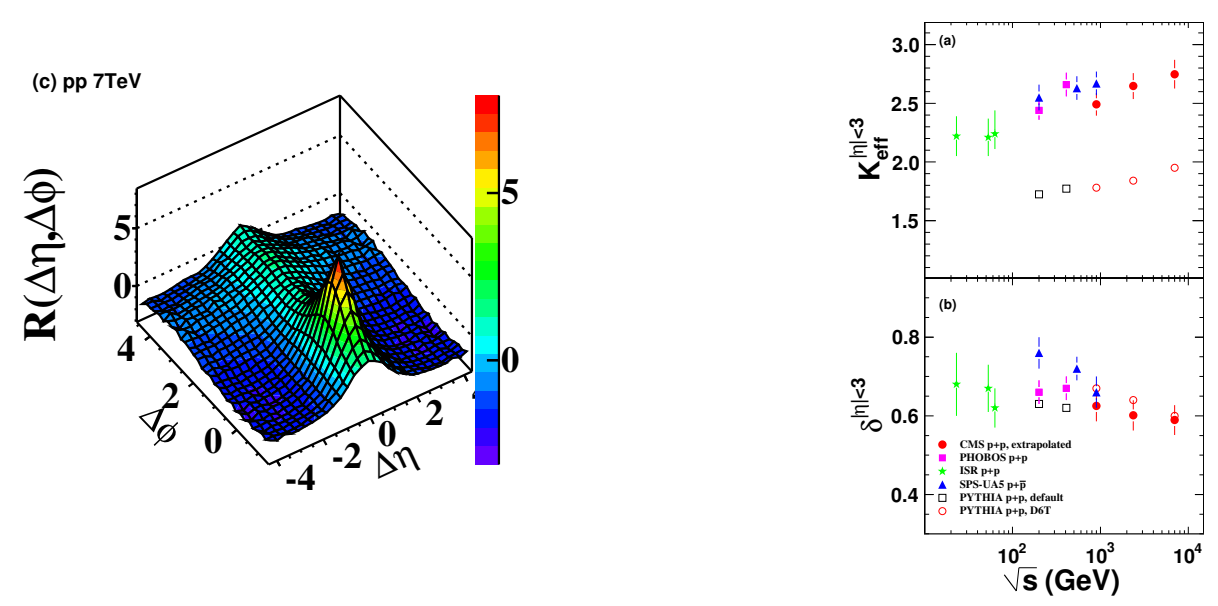

Figure 1: Two-particle angular correlation Figure 2: (a) $K_{\text {eff }}$ and (b) $\delta$ as a function of $\sqrt{s}$ using a model function in $\Delta \eta$ and $\Delta \phi$ in pp collisions at dependent extrapolation of CMS data to $p_{T}=0$ and $|\eta|<3$ $\sqrt{s}=7 \mathrm{TeV}$. as well as PHOBOs, UA5 and ISR. PYTHIA results with two different tunes are also shown.

\section{Bose-Einstein Correlations}

In particle collisions, the space-time structure of the hadronization source can be studied using measurements of Bose-Einstein correlations (BEC) between pairs of identical bosons. Constructive interference affects the joint probability for the emission of a pair of identical bosons with four-momenta $p_{1}$ and $p_{2}$. Experimentally, the proximity in phase space between final-state particles is quantified by the quantity $Q=\sqrt{-\left(p_{1}-p_{2}\right)^{2}}=\sqrt{M^{2}-4 m_{\pi}^{2}}$, where $M$ is the invariant mass of the two particles, assumed to be pions with mass $m_{\pi}$. The BEC effect is observed as an enhancement at low $Q$ of the ratio of the $Q$ distributions for pairs of identical particles in the same event, and for pairs of particles in a reference sample that by construction is expected to include no BEC effect:

$$
R(Q)=(d N / d Q) /\left(d N_{r e f} / d Q\right)
$$


which is then fitted with the parameterization $R(Q)=C[1+\lambda \Omega(Q r)] \cdot(1+\delta Q) . \Omega$ is often phenomenologically parameterized as $\Omega(Q r)=e^{-Q r}$ or $\Omega(Q r)=e^{-(Q r)^{2}}$ [4]. The parameter $\lambda$ measures the strength of BEC for incoherent boson emission from independent sources, $\delta$ accounts for long-distance correlations, and $C$ is a normalization factor.

All pairs of same-charge particles with $Q$ between 0.02 and $2 \mathrm{GeV}$ collected at 0.9 and 2.36 $\mathrm{TeV}$ are used for the measurement [5]. Different methods are designed to pair uncorrelated charged particles and to define reference samples used to extract the distribution in the denominator of Eq. (3.1). Opposite-charge pairs: this data set is a natural choice but contains resonances $(\eta, \rho$, ...) which are not present in the same-charge combinations. Opposite-hemisphere pairs: tracks are paired after inverting the three-momentum of one of the two particles; this procedure is applied to pairs with same and opposite charges. Rotated particles: particle pairs are constructed after inverting the $p_{t}$ of one of the two particles. Pairs from mixed events: particles from different events are combined with the following methods: i) events are mixed at random; ii) events with similar charged particle multiplicity in the same $\eta$ regions are selected; iii) events with an invariant mass of all charged particles similar to that of the signal are used to form the pairs.

In order to reduce the bias due to the construction of the reference samples, a double ratio $\mathscr{R}$ is defined as $\mathscr{R}(Q)=R / R_{M C}=\left(\frac{d N / d Q}{d N / d Q_{r e f}}\right) /\left(\frac{d N / d Q_{M C}}{d N / d Q_{M C, r e f}}\right)$, where $Q_{M C}$ and $Q_{M C, \text { ref }}$ refer to the $Q$ distributions from the default simulation, which does not include a modeling of Bose-Einstein correlations. As none of the definitions of the reference samples is preferable a priori, an additional, "combined" double ratio $\mathscr{R}^{\text {comb }}$ is formed, where the data and MC distributions are obtained by summing the $Q$ distributions of the seven corresponding reference samples.

The distributions $\mathscr{R}^{\text {comb }}$ for 0.9 and $2.36 \mathrm{TeV}$ data show a clear enhancement at low $Q$ as it can be seen in Fig. 3. The parameterization $\Omega(Q r)=e^{-Q r}$ and $\Omega(Q r)=e^{-(Q r) 2}$ is used to fit $\mathscr{R}^{\text {comb }}$. Only the exponential form is found to fit reasonably the data and the BEC parameters measured with the combined reference sample are $\lambda=0.625 \pm 0.021$ (stat.) \pm 0.046 (syst.) and $r=1.59 \pm 0.05$ (stat.) \pm 0.19 (syst.) fm at $0.9 \mathrm{TeV} ; \lambda=0.663 \pm 0.073$ (stat.) \pm 0.048 (syst.) and $r=$ $1.99 \pm 0.18$ (stat.) \pm 0.24 (syst.) fm at $2.36 \mathrm{TeV}$. A significant increase of $r$ with the charged-particle multiplicity and a slight decrease of $\lambda$ are observed as shown in Fig.3 (right). These results agree with what has been measured by previous experiments [6].

\section{Conclusion}

CMS has measured two-particle angular correlations over a wide range in $\Delta \eta$ and $\Delta \phi$ in pp collisions at $\sqrt{s}=0.9,2.36$ and $7 \mathrm{TeV}$. Short-range correlations are observed over the full range in $\Delta \phi$, with a maximum at $\Delta \eta=0$ which becomes wider at larger $\Delta \phi$. In the context of the cluster model, the effective cluster size and decay width are extracted from the two-particle pseudorapidity correlation function, and compared with previous experiments, as well as PYTHIA event generator. Dependence of the cluster size on beam energy is observed, while the cluster width is essentially constant. Recently, analysis on long-range $(2.0<|\Delta \eta|<4.8)$ correlations with high multiplicity events at $\sqrt{s}=7 \mathrm{TeV}[2]$ has shown a ridge-like structure at the near-side $(\Delta \phi \approx 0)$, resembling similar features observed in heavy-ion experiments [7, 8].

Bose-Einstein correlations have been measured for the first time at the LHC by the CMS experiment in $p p$ collisions at 0.9 and $2.36 \mathrm{TeV}$ center-of-mass energies. The main systematic 

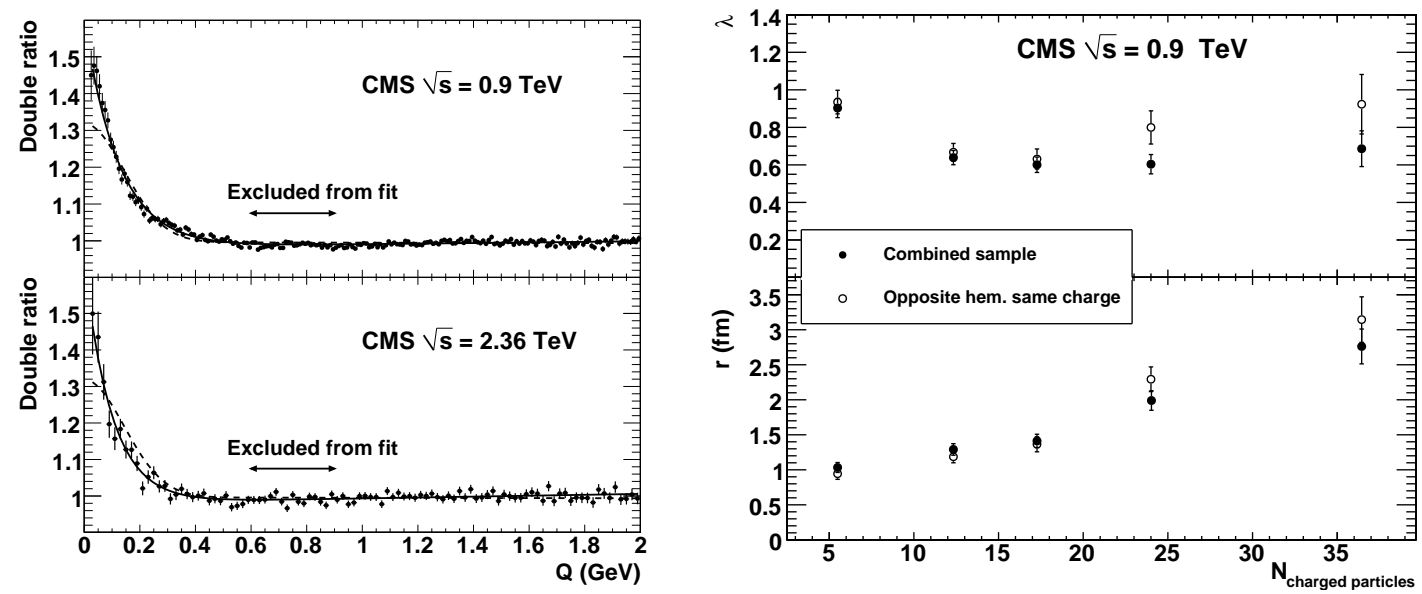

Figure 3: Left: $\mathscr{R}^{c o m b}$ distributions fitted with exponential (full line) and Gaussian (dashed line) functions. Right: $\lambda$ (top ) and $r$ (bottom) parameters as function of the charged-particle multiplicity at $0.9 \mathrm{TeV}$ for the combined (dots) and Opposite-hemisphere same charge (open circles) reference samples.

issue affecting BEC measurements was studied through the use of multiple reference samples to extract the signal. We have observed, for the first time with this degree of significance, and for all reference samples, that the shape of the signal is not described by a Gaussian function, but rather by exponential or more complex functions. An increase of the effective size of the emission region with charged-particle multiplicity, disputed for a long time [6], is now very clearly observed in $p p$ collisions with a single experiment.

\section{References}

[1] CMS Collaboration, The CMS experiment at the CERN LHC, JINST 3 (2008) S08004.

[2] CMS Collaboration, Observation of long-range, near-side angular correlations in proton-proton collisions at the lhc, JHEP 2010 (2010) 1-38.

[3] A. Morel and G. Plaut, How Do Clusters Look in Semiinclusive Cross-Sections?, Nucl. Phys. B78 (1974) 541 .

[4] G. Kozlov, O. Utyuzh, G. Wilk, and Z. Wlodarczyk, Some forgotten features of Bose-Einstein Correlations, Phys. Atom. Nucl. 71 (2008) 1502.

[5] CMS Collaboration, First Measurement of Bose-Einstein Correlations in Proton-Proton Collisions at $\sqrt{s}=0.9$ and $2.36 \mathrm{TeV}$ at the LHC, PRL 105 (Jul, 2010) 032001.

[6] W. Kittel and E.A. De Wolf, Soft Multihadron Dynamics. World Scientific, Singapore 2005.

[7] PHOBOS Collaboration, System size dependence of cluster properties from two-particle angular correlations in $c u+c u$ and $a u+a u$ collisions at $\sqrt{s_{N N}}=200$ gev, Phys. Rev. C 81 (Feb, 2010) 024904.

[8] STAR Collaboration, Three-particle coincidence of the long range pseudorapidity correlation in high energy nucleus-nucleus collisions, PRL 105 (Jul, 2010) 022301. 